\title{
Towards disentangling photodesorption and photodissociation in astronomical ice analogues
}

\author{
Michał Bulak ${ }^{1}$ (D), Daniel Paardekooper ${ }^{2}$, Jordy Bouwman $^{1}{ }^{(D)}$, \\ Gleb Fedoseev ${ }^{1}$ and Harold Linnartz ${ }^{1}$ (D) \\ ${ }^{1}$ Laboratory for Astrophysics, Leiden Observatory, Leiden University, PO Box 9513, 2300 RA \\ Leiden, the Netherlands \\ email: bulak@strw.leidenuniv.nl \\ ${ }^{2}$ Jet Propulsion Laboratory, California Institute of Technology, Pasadena, California, US
}

\begin{abstract}
UV irradiation of ices plays an important role in different inter- and circumstellar environments. Following the absorption of UV photons in ice, two processes compete: photodesorption and photodissociation/chemistry. From an experimental point of view it is very hard to discriminate between photodesorption and photodissociation (and resulting photochemistry). In this work we present our first attempts to distinguish both effects. The performance is demonstrated on the example of CO-ice, known not to dissociate upon UV irradiation, and $\mathrm{CH}_{4}$-ice that does fragment.
\end{abstract}

Keywords. astrochemistry, methods: laboratory, techniques:molecular processes, ISM: molecules

\section{Introduction}

In cold regions of the Interstellar Medium (10-20 K), ice mantles are the source of chemical diversity. At the same time, gas-phase formation rates have been shown to be not sufficient to explain astronomical abundances of molecules like water (Bergin et al. (1995)), methanol (Geppert et al. (2006)) and several larger COMs. In order to explain the observed gas-phase abundances, a non-thermal mechanism is necessary that transfers molecules effectively and 'in one piece' from solid-state to gas-phase (e.g. $\mathrm{CH}_{3} \mathrm{OH}$, Guzman et al. (2013)).

The non-thermal desorption mechanism that we focus on here is UV photon stimulated desorption. However, ice UV processing also initiates dissociation (Bertin et al. (2016)). A challenge associated with quantifying UV processes in ices is disentangling photodesorption and photo-dissociation/chemistry. The new concept introduced here is based on a technique developed a few years ago in which the vacuum UV irradiation of ices was characterized by laser induced desorption combined with time-of-flight mass spectrometry (Paardekooper et al. (2014)). Here, we extend on that technique, focusing on pure CO and $\mathrm{CH}_{4}$ ices, as test environments; work dealing with $\mathrm{CH}_{3} \mathrm{CN}$ is currently in preparation.

\section{Methods}

Ice analogues are grown in an Ultra High Vacuum (UHV) apparatus, MATRI2CES. To track molecular abundances in the ices, laser-desorbed species are ionized using an electron gun and accelerated into the time-of-flight mass spectrometer. Electron impact ionization induces fragmentation of molecular ions, which is taken into account. Broadband vacuum UV light is generated by a Microwave Discharge Hydrogen Lamp 


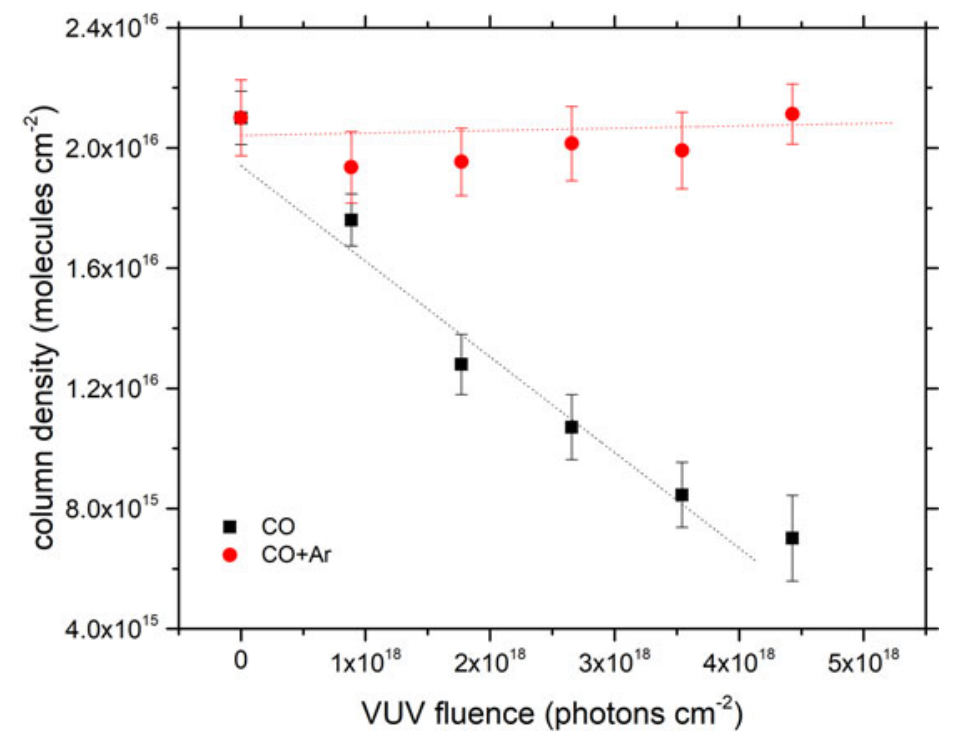

Figure 1. Integrated intensities of mass peaks representing CO.

(115-170 nm) with a typical flux of $10^{14}$ photons $\mathrm{cm}^{-2} \mathrm{~s}^{-1}$. Ice thickness and photon flux are measured using laser interferometry and a photodiode. Typical values amount to respectively 20-50 ML and $10^{18}$ photons $\mathrm{cm}^{-2}$. For each ice $\left(\mathrm{CO}\right.$ and $\left.\mathrm{CH}_{4}\right)$, two experiments are performed. The experiments probe the different effects of UV photoprocessing of a pure ice with and without a top layer of Argon. Argon's primary role is to quench photodesorption, therefore, the difference between experiments, independent of the photo-dissociation/chemistry, gives the total photodesorption yield. Argon does not interfere with the ice's photochemistry, and does not absorb UV light.

\section{Results}

Photodesorption of carbon monoxide: Experiments with $\mathrm{CO}$ are performed in order to test the new method against photodesorption values available in literature. CO does not dissociate, therefore the decrease in the $\mathrm{CO}$ signal can be directly associated with photodesorption. Figure 1 shows $\mathrm{CO}$ abundance in experiments with and without $\mathrm{Ar}$ coating. The photodesorption rate was calculated to be $(3.2 \pm 0.3) \times 10^{-3}$ mol. photon ${ }^{-1}$, which agrees with previous studies: e.g. $(2.7 \pm 1.3) \times 10^{-3}$ mol. photon ${ }^{-1}$ in Öberg et al. (2009b).

Photodesorption of methane: In case of $\mathrm{CH}_{4}$, analysis is more complex as methane ice also photodissociates. It produces radicals $\left(\mathrm{CH}, \mathrm{CH}_{2}, \mathrm{CH}_{3}\right)$ which recombine to form bigger hydrocarbons, mainly $\mathrm{C}_{2} \mathrm{H}_{2}, \mathrm{C}_{2} \mathrm{H}_{4}, \mathrm{C}_{2} \mathrm{H}_{6}$ (Bossa et al. (2015)). In this study we find a preliminary photodesorption rate value of $(3.1 \pm 0.5) \times 10^{-2}$ mol. photon $^{-1}$, which is higher than in (Dupuy et al. (2017)). We are now looking into reasons for this.

We expect that this approach holds potential to derive photodesorption rates of larger COMs, for which dissociation plays an important role.

\section{References}

Bergin, E. A. and Langer, W. D. and Goldsmith, P. F. 1995, ApJ, 441, 222

Bertin, M., Romanzin, C., Doronin, M., et al. 2016, ApJ, 817, 2

Bossa, J. B., Paardekooper, D. M., Isokoski, K., et al. 2015 PCCP, 17, 26 
Dupuy, R., Bertin, M., Féraud, G., et al. 2017, A\& A, 603, A61

Geppert, W. D., Hamberg, M., Thomas, R. D., et al. 2006, Faraday Discussions, 133, 177

Guzmán, V. V., Goicoechea, J. R., Pety, J., et al. 2013, A\&A, 560, A73

Öberg, K. I. and van Dishoeck, E. F. and Linnartz, H. 2009b, A\&A, 496, 281

Paardekooper, D. M. and Bossa, J. B. and Isokoski, K., et al. 2014, RSI, 85, 10 